\title{
Opening Windows into the Cell: Bringing Structure to Cell Biology using Cryo-Electron Microscopy
}

Elizabeth Villa ${ }^{1}$, Reika Watanabe ${ }^{1}$, Robert Buschauer ${ }^{1,2}$, Kanika Khanna ${ }^{1}$, Vinson Lam ${ }^{1}$

${ }^{1}$. Division of Biological Sciences, University of California San Diego, La Jolla, USA.

2. Current Address: Gene Center, Ludwig Maximillian University, Munich, Germany.

Single-particle cryo-electron microscopy has established itself as a central tool in structural biology, ideally suited to study large macromolecular complexes from in vitro samples. It stands today at the center of integrative modeling, where cryo-EM maps serve as a scaffold to combine data from different sources. Cryo-electron tomography (CET) can extend the applicability of cryo-electron microscopy to study these complexes in situ. Recent technological advances allow CET to be applied to questions in cell biology that go beyond structural determination, where a quantitative analysis of cellular features is required including molecular distribution and supramolecular architecture. However, the ability of CET to provide threedimensional landscapes of cells is limited by the thickness of the sample that can be examined in situ with a resolution that is high enough for the identification of molecular species. For intermediate resolution electron microscopes, samples must be of less than $500 \mathrm{~nm}$ in thickness. Most cells, specially eukaryotes, exceed the thickness accessible to CET, limiting its routine application to isolated or reconstituted subcellular systems, small prokaryotic cells, or thin peripheral regions of cells.

To overcome such limitation, focused-ion-beam milling at cryo temperatures (cryo-FIB) can produce samples of adequate thickness for CET from frozen-hydrated biological cells. This novel approach is applicable to all types of cells grown or deposited directly on EM grids by generating 100-400 nm lamellae that are supported by the surrounding cellular material, and thus remain in the EM grid. The grid is then transferred out of the dual beam and stored at liquid nitrogen temperatures for subsequent imaging in a cryo-TEM. This workflow, combined with correlative fluorescence microscopy, holds the promise of serving as a bridge between cellular and molecular scales. The routine use of this methodology will be described in detail, along with its application to the study of the structure of macromolecular complexes that were previously inaccessible to structural studies in their native environment inside the cell, including chromatin and proteins involved in Parkinson's disease. 